\title{
Desarrollo del pensamiento crítico a través de la competencia comunicativa en la educación superior: una propuesta reflexiva
}

\author{
Development of critical thinking through communicative skill in higher \\ education: a reflective proposal
}

\author{
M. Carme Balaguer Fábregas ${ }^{1}$, Maria Pujol Valls ${ }^{2}$, Mariona Graell Martín ${ }^{3}$ \\ ${ }^{1}$ Universidad Internacional de Cataluña, España (mcbalaguer@uic.es) \\ ${ }^{2}$ Universidad Internacional de Cataluña, España (mpujolv@uic.cat) \\ ${ }^{3}$ Universidad Internacional de Cataluña, España (mgraell@uic.es)
}

Recibido el 30 de noviembre de 2017; revisado el 15 de mayo de 2018; aceptado el 18 de mayo de 2018 ; publicado el 1 de junio de 2018

\section{RESUMEN:}

El objetivo de este artículo es presentar un programa de investigación transversal para analizar el desarrollo del aprendizaje de los estudiantes que cursan la formación inicial de maestros de Educación Infantil. A lo largo de la carrera los estudiantes tienen que adquirir diferentes competencias que les permitan convertirse en buenas personas y buenos profesionales. Pero ¿cuál es el proceso a través del que los estudiantes pasan a asumir el pensamiento crítico y las competencias comunicativas que les permitirán ser maestros? ¿Cuál es la forma como los estudiantes autorregulan sus aptitudes lingüísticas, orales y escritas? ¿Son conscientes y críticos con su propio aprendizaje en esta materia? En este proyecto se quiere observar el proceso que nuestros estudiantes siguen para desarrollar estas cuestiones. A través de procesos reflexivos, se plantea el estudio de los procesos metacognitivos que permiten la cristalización de los conocimientos y las habilidades lingüísticas. Se prevé una investigación durante un período de 2 años. La metodología que se va a implementar es un modelo longitudinal mixto (cualitativo, cuantitativo) que se espera aporte informaciones sobre la percepción de los estudiantes de grado de Educación Infantil, acerca el desarrollo de su competencia lingüística y comunicativa. En este artículo concretamente se pretende describir el diseño de la investigación, el instrumento elaborado y los primeros resultados de naturaleza cualitativa de los que se desprende la necesidad de mejorar los procesos de reflexión de los estudiantes de grado de Educación Infantil.

PALABRAS CLAVE:

FORMACIÓN INICIAL DE MAESTROS, PENSAMIENTO CRÍTICO, COMPETENCIA COMUNICATIVA, ENSEÑANZA REFLEXIVA

\begin{abstract}
:
The aim of this article is to present a cross-sectional research program for a preservice education degree. Its goal is to analyse the learning development of the students that attend the preservice teacher education for pre-school teachers. Throughout their degree, students should be trained in different skills that allow them to become good people and good professionals. But, what is the process through which students acquire critical thinking and communication skills that will enable them to become teachers? In what way do students selfregulate their linguistic, oral and written skills? Are they aware and critical of their own learning in this area? In this project we want to observe the process that our students follow to develop these issues. For this reason, the study of metacognitive processes that allow the crystallization of knowledge and linguistic skills is considered. This analysis is obtained through reflective processes. An investigation is planned for a period of 2 years. The methodology that will be implemented is a mixed
\end{abstract}


longitudinal model (qualitative, quantitative). It is expected to provide information on the perception of students of Early Childhood Education on the development of their linguistic and communicative competence. In this article, we intend to describe the design of the research, the instrument developed and the first results of qualitative nature. Finally, the need to improve the reflection processes of the students of the Early Childhood Education degree is revealed from results obtained.

KEYWORDS: INITIAL TEACHER TRAINING; CRITICAL THINKING, COMMUNICATIVE SKILL; REFLECTIVE TEACHING

\section{INTRODUCCIÓN}

La importancia de la reflexión sobre el contenido de los grados de formación inicial de estudiantes de educación y su relación con las experiencias prácticas es uno de los ejes para la adquisición de competencias profesionales (Puig, 2009; Schön, 1992). Además, es una evidencia que la reflexión sobre los propios procesos de aprendizaje y las estrategias personales de adquisición de conocimientos también constituyen un referente que ayuda en la formación como docente (Domingo Roget, 2013). Estas reflexiones han propiciado el desarrollo de esta investigación, que se encuentra en una fase inicial, y de la cual se presentan en este artículo el diseño de la investigación y unos resultados preliminares recogidos en el ámbito cualitativo.

Las propuestas de enseñanza y aprendizaje consciente (Monereo y Badia, 2011) ponen en consideración la necesidad de dotar a los estudiantes de educación de los mecanismos necesarios para aprovechar sus experiencias como alumnos y usarlas como referencia en los nuevos contenidos didácticos desarrollados en los grados de educación. Desde la Facultad de Educación de la Universitat Internacional de Catalunya, se ha diseñado un programa de mejora de los procesos reflexivos de los estudiantes, vinculando estos procesos con la competencia comunicativa tanto oral como escrita. Concretamente este proyecto se ha desarrollado en las asignaturas de Enseñanza y Aprendizaje de la Lengua y la Lectoescritura I, II, III y IV.

Los objetivos propuestos son los siguientes: 1. Evidenciar el conocimiento de los procesos de enseñanza y aprendizaje del lenguaje y la lectoescritura; 2. Argumentar de manera crítica el desarrollo del propio proceso de enseñanza y aprendizaje, y 3. Mostrar iniciativa para profundizar en los contenidos estudiados.

Los antecedentes teóricos que sirven de referentes en esta investigación se sitúan en los psicólogos interaccionistas y culturales que han investigado sobre la importancia del lenguaje en los procesos de aprendizaje, así como los teóricos del aprendizaje estratégico focalizado en la consciencia del aprendizaje y de la metacognición.

Dentro de los procedimientos de reflexión se ha fundamentado esta investigación en las aportaciones de Domingo Roget (2013); Páez y Puig (2013); Rubio Hurtado, Galvan Fernández y Rodríguez Illera (2013); Schön (1992) y Vilà Baños y Aneas (2013).

La práctica reflexiva $(\mathrm{PR})$ es una metodología de formación en la que los principales elementos para iniciarla son las experiencias de docentes y estudiantes en formación inicial de maestro, por lo tanto, parte antes de la persona que del saber teórico.

Este modelo permite, además de profundizar en el conocimiento de la materia, en la didáctica y en la pedagogía, que el profesorado se haga consciente de su práctica docente y convierta la reflexión en la práctica y sobre la práctica en un hábito que integrará en su quehacer diario. El procedimiento es el análisis de la propia acción docente, reflexionar sobre ella, compartir las reflexiones y construir conjuntamente propuestas de mejora (Páez y Puig, 2013).

La necesidad e importancia de compartir las reflexiones sugieren que la mejor manera de trabajar la práctica reflexiva es en grupos reducidos, conducidos por un experto, aunque dominados por relaciones comunicativas simétricas.

Estos grupos reducidos pasaran a considerarse comunidades de aprendizaje donde impera un clima de confianza para que los participantes puedan construir conocimiento, a partir de ciclos sistemáticos de reflexión. En este sentido, el principal objetivo de la práctica reflexiva es transformar la práctica diaria en un proceso de investigación-acción con finalidad de mejora sobre esta misma práctica (Bisquerra Alzina, 2004; Domingo Roget, 2013).

\section{MATERIAL Y MÉTODO}

Al situar esta investigación en una primera fase, tal como se ha determinado anteriormente, el método de investigación desarrollado ha sido un método cualitativo. Se han analizado y categorizado las reflexiones de una muestra de 19 estudiantes que cursaban $2^{\circ}$ curso del grado de Educación Infantil y 
dentro del marco de la asignatura Aprendizaje de la Lengua y la Lectoescritura II. Concretamente, estas reflexiones se han extraído de los portafolios de aprendizaje desarrollados durante el curso 20162017. La extracción de datos cualitativos, en este caso, se ha realizado a partir de un proceso de teoría fundamentada. Los procedimientos seguidos son los siguientes: elaboración de un corpus conceptual donde se recogían todas las evidencias; lectura y categorización de los contenidos; análisis de los resultados.

El instrumento utilizado para recoger las experiencias ha sido un diseño de portafolio de aprendizaje, elaborado en base a las competencias transversales y de titulación determinadas en las guías docentes de las asignaturas de Aprendizaje de la Lengua y la Lectoescritura de los grados de Educación Infantil. Estas competencias son las siguientes: comunicarse claramente y de forma correcta en la lengua de enseñanza, de forma oral y escrita según el nivel C1 de acuerdo con el Marco Común Europeo de Referencia para las Lenguas; ser experto en la materia impartida y en los métodos de enseñanza: poseer los conocimientos y habilidades suficientes acerca de la materia que se enseña y saber qué métodos didácticos tiene que aplicar para crear un gran entorno de aprendizaje, donde los alumnos puedan aprender el bagaje cultural que necesitan para vivir en sociedad -y lograrlo de una manera contemporánea, profesional y sistemática-; fomentar su responsabilidad en lo que se refiere a su desarrollo profesional: analizar, reflexionar $\mathrm{y}$ desarrollar sus puntos de vista sobre la profesión y su competencia como maestro, saber explicitarlos y saber cómo ponerse al día.

Las condiciones para la elaboración del portafolio de aprendizaje son las siguientes: recoger el material docente aportado por las profesoras de las distintas asignaturas (bibliografía, documentos y PowerPoint elaborados por las profesoras, etc.); recoger los apuntes tomados por cada estudiante; incluir las actividades formativas realizadas en las clases prácticas y en los diferentes trabajos grupales; aportar de manera voluntaria ampliaciones relacionadas con la temática desarrollada; aportar una reflexión personal argumentada de cada tema; aportar una reflexión personal final relativa a las percepciones, aprendizajes y sentimientos emergidos en el curso de cada asignatura.

En relación a los procesos de reflexión, se ha seguido el modelo R5 (Domingo Roget, 2013) que se reproduce en la figura 1, aunque adaptando sus fases a un entorno de formación universitaria.

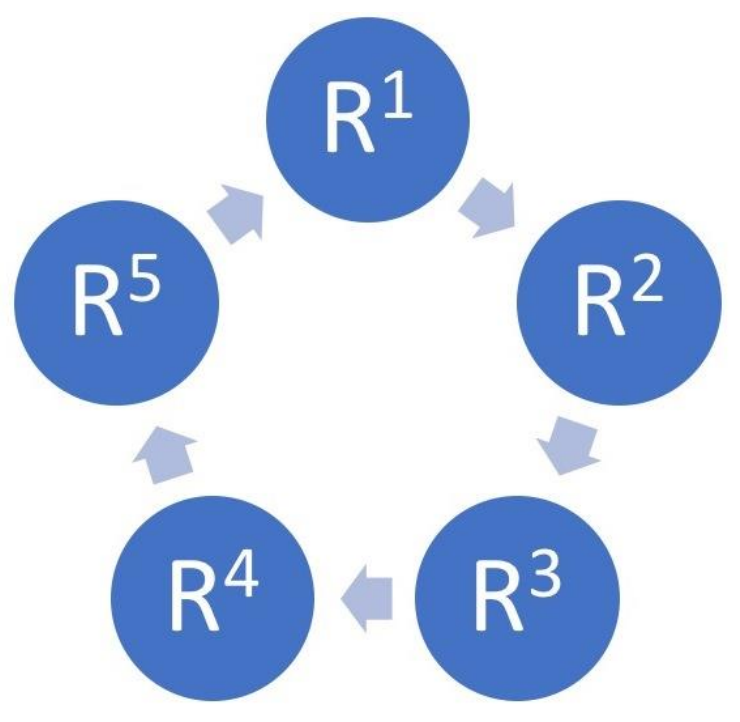

Figura 1: Fases del Método R5 (Domingo Roget, 2013, pág. 269)

Según esta autora, el circuito de reflexión se inicia con la selección de una experiencia ocurrida en el aula (R1), que en el entorno universitario se ha considerado como el uso de una metodología, la percepción de distintos aprendizajes o cualquier elemento que los estudiantes puedan considerar relevante. Seguidamente, en la fase R2, la experiencia escogida se tiene que reconstruir de manera verbal (oral o por escrito). En la fase R3 se produce una reflexión individual, tomando como referencia el conocimiento de la materia desarrollada, su relación con el contexto y la decisión sobre qué aspectos convendría modificar para mejorar el aprendizaje.

Las fases R4 y R5 se refieren a compartir las conclusiones de la reflexión individual. Concretamente en R4, se han propiciado espacios de participación para intercambiar las aportaciones individuales, y en la fase R5 se ha solicitado un relato sobre cómo se han integrado los aprendizajes surgidos de la interacción reflexiva en su percepción como futuro docente.

Los resultados de aprendizaje que se esperan de esta experiencia también se han vinculado con los propuestos en las respectivas guías docentes. Son los siguientes: conoce la incidencia de la interacción entre iguales $\mathrm{y}$, entre niños y adultos en los procesos pedagógicos como el trabajo cooperativo, el individual y el colectivo; conoce los procesos mentales a seguir para desarrollar un análisis exhaustivo de los casos a estudiar; reconoce la necesidad de esforzarse individualmente para conseguir alcanzar los objetivos de la comunidad educativa. 


\section{RESULTADOS}

Como se ha comentado anteriormente, en este artículo se pretenden describir los resultados conseguidos gracias a una investigación cualitativa, que se corresponden con una primera fase de esta investigación transversal.

La categorización del corpus de conocimiento extraído de las evidencias ha favorecido la elaboración de las categorías emergentes de las distintas muestras de reflexión. Esto se ha conseguido determinando diferentes unidades de significado recurrentes. Para esta categorización, se ha seguido un procedimiento inductivo de análisis de casos para construir la teoría.

De las 19 pruebas de reflexión analizadas, se han hallado 46 unidades conceptuales que se han agrupado en las categorías que aparecen en la tabla 1.

Tabla 1. Categorías conceptuales

\begin{tabular}{ccc}
\hline Categorías emergentes & Frecuencia & $\%$ \\
\hline Funcionalidad/utilidad & 19 & $41,3 \%$ \\
Resumen de contenidos & 8 & $17,3 \%$ \\
Proceso de aprendizaje & 7 & $15,2 \%$ \\
Interés por la materia & 4 & $8,65 \%$ \\
Crítica de la asignatura & 3 & $6,5 \%$ \\
Agradecimientos & 2 & $4,3 \%$ \\
Autocrítica & 2 & $4,3 \%$ \\
Relación con otras teorías & 1 & $2,1 \%$ \\
\hline Total & 46 & $100 \%$
\end{tabular}

La categoría de Funcionalidad/utilidad se refiere a la utilidad de los conocimientos que los estudiantes han adquirido; en estos casos, a menudo se destaca la utilidad de los aprendizajes para la profesionalización. La de Resumen de contenidos se aplica a las unidades conceptuales que demuestran que los estudiantes a veces confunden la reflexión sobre la asignatura con un resumen de sus contenidos; por lo tanto, esta categoría se adjudica a los textos que supuestamente son reflexivos, pero en realidad solo exponen una síntesis de la materia trabajada. La de Proceso de aprendizaje se relaciona con los comentarios de los estudiantes sobre su propio aprendizaje; así pues, reflexionan sobre el logro de los objetivos y competencias que aparecen en la guía docente de la asignatura, con lo que manifiestan habilidades de autoregulación (metacognición). La de Interés por la materia se detecta cuando los estudiantes expresan motivación intrínseca o extrínseca por la asignatura; a veces, en el caso de la motivación extrínseca, esta categoría coincide con la categoría de funcionalidad ya que los estudiantes expresan su interés por la materia porque ven en ella una utilidad futura. La de Crítica de la asignatura es relevante para las reflexiones que incluyen juicios positivos o negativos de la asignatura; estas valoraciones tanto pueden aportar sugerencias de mejora como opiniones elogiosas relacionadas sobre todo con la categoría de Funcionalidad/utilidad. La de Agradecimientos hace referencia a los comentarios para gratificar a la profesora; en algunos portafolios, los estudiantes dan las gracias por haber superado ciertos desconocimientos que les provocaban inseguridad o angustia. La de Autocrítica se entiende como la valoración que los estudiantes efectúan sobre su actuación; estas consideraciones a veces están relacionadas con las de la categoría Crítica de la asignatura. Finalmente, la de Relación con otras teorías es presente en las reflexiones que demuestran una transferencia de los aprendizajes entre asignaturas; es decir, para construir su discurso sobre Enseñanza y Aprendizaje de la Lengua y la Lectoescritura II, aportan conocimientos adquiridos en otras asignaturas.

\section{DISCUSIÓN}

La designación de las categorías aparecidas en los resultados induce a pensar en algunos elementos presentes en las percepciones de los estudiantes. En primer lugar, se destaca que mayoritariamente los estudiantes valoran la funcionalidad de los aprendizajes, con expresiones al estilo de "querría resaltar que los temas tres y cuatro son los que he encontrado más interesantes y útiles ya que los he podido relacionar, tanto con mis prácticas anteriores en diferentes escuelas, como con mi futura práctica como docente".

La evidente vinculación que hacen los estudiantes de la funcionalidad de los aprendizajes con el interés que les suscitan en el momento en que son cursados coincide con las teorías de la motivación vinculadas con la funcionalidad (Woollfolk, Hughes y Walkup, 2008; Alonso Tapia y Montero García-Celay, 2008). Estos autores coinciden en la planificación de actividades funcionales para garantizar la motivación. En el ámbito universitario, se puede relacionar la motivación con el diseño de las actividades formativas concretadas en el análisis de casos documentados. En general, los estudiantes valoran el estudio de casos como actividad formativa por la aproximación que se hace de la teoría a la realidad. También suele ser recurrente que se trata de una actividad que inicialmente sugiere dificultad por cuanto los estudiantes no están habituados a 
desarrollar. Su interés crece con la percepción de su competencia y la atribución de éxito en su resolución (Miras, 2008).

Un comentario también interesante es que, en la mayoría de las reflexiones sobre la funcionalidad de los aprendizajes, aparece la reflexión sobre la prospectiva profesional, lo que nos refuerza que se están desarrollando las competencias de titulación. Según Huertas, Alonso Tapia, Trías, Ardura y García (2011), las identidades de los estudiantes universitarios presentan variaciones motivacionales según la situación o el contexto en el que se encuentran. El contexto que se describe en este artículo es el de unas asignaturas trascendentes en relación a los objetivos de aprendizaje del alumnado de Educación infantil. Concretamente se enfrentan a la didáctica de la lectoescritura inicial. Las percepciones de las personas, las reglas sociales, los estereotipos en relación a la lectoescritura determinan una motivación añadida a los estudiantes universitarios que va modificando $y$ responsabilizando su identidad docente en un futuro próximo (Huertas et al, 2011).

Un elemento preocupante es el porcentaje elevado en los resultados de personas que confunden la reflexión con el resumen de contenidos. Cabría pensar en el análisis de estos conceptos a partir de un proceso de indagación y práctica (Le Fevre, 2010) que permitiría la interiorización y distinción de los dos tipos de prácticas: el resumen y la reflexión.

Desde otro punto de vista argumental, la categoría Resumen de contenidos también se podría vincular con la categoría Proceso de aprendizaje por cuanto los estudiantes parafrasean los contenidos que ellos consideran que han interiorizado en su proceso de aprendizaje, pero los verbos no mentalistas (Gallardo-Paúls, 2008) que se muestran determinan que es una selección sin reflexión. Es decir, se trata de un listado de contenidos. Un ejemplo de comentario relacionado con esta categoría es el siguiente: "Así que podemos decir que la lectoescritura es la habilidad que nos permite plasmar el lenguaje, hacerlo permanente y accesible sin límites. Nos da información y formación, y así, nos permite avanzar en nuevos conocimientos y saberes, además, hacemos descubrimientos sorprendentes." O incluso se han encontrado ejemplos más teóricos, como el siguiente: "Hay diferentes elementos que hay que tener en cuenta con relación al lenguaje: En primer lugar, la comprensión del mensaje, es decir, es aquella capacidad que permite a la persona entender lo que otra persona le está explicando, informando o sugiriendo a través de la vista y la escucha. En la comprensión del lenguaje, se convierten en claves multitud de habilidades cognitivas que facilitarán al niño la comprensión del mensaje del emisor, entre ellas destacamos los procesos cognitivos $\mathrm{y}$ paralelamente los procesos metacognitivos".

La categoría correspondiente al reconocimiento de los procesos de aprendizaje seguidos no ha sido suficientemente evidenciada. Los comentarios relativos a la construcción del conocimiento a través del desarrollo de objetivos y contenidos son como el siguiente: "Al haber compartido una asignatura el año anterior con nosotros, ya sabía más o menos las diferentes metodologías que se utilizarían y cómo íbamos a construir el proceso de aprendizaje. Estas fueron diversas: clases magistrales, trabajos en grupo, presentaciones, entrevistes grupales, entre otras. Creo que todas las metodologías han sido bastante acertadas, me han ayudado a lograr y comprender mejor los aprendizajes y a construirlos día a día".

Destacamos que se toma consciencia de la necesidad de "construir" el conocimiento, de utilizar para ello metodología variada, de hacer diferente tipo de agrupaciones. Implícitamente están reconociendo un proceso constructivista de enseñanza y aprendizaje, que en definitiva es el modelo de aprendizaje que se intenta transmitir (Bruner, 1997; Vila Mendiburu, 1999; Vygotsky, 2012).

El interés de la materia ha sido otra categoría de la que se desprenden dos acepciones. En primer lugar, el interés se ha vinculado en muchas ocasiones a la funcionalidad futura, tema que ya se ha comentado anteriormente. En segundo lugar, el interés también se ha manifestado con relación al gusto por el contenido intrínseco de la materia (Miras, 2008). Esto se refleja en aportaciones como la siguiente: "Este es uno de los temas que personalmente me ha gustado más, lo he encontrado muy y muy interesante. Nos habla de que, cuando los niños empiezan a leer, ellos empiezan a activar una serie de procesos cognitivos que les sirven para poder comprender un fragmento de texto o todo un texto entero".

También se han encontrado comentarios críticos sobre la asignatura. Los negativos mayoritariamente se han referido a algunos aspectos de la metodología. Todas estas aportaciones son objeto de reflexión docente con el objetivo de mejorar para un nuevo curso (Domingo Roget, 2013). Las críticas han sido semejantes a estas: "Para finalizar, me gustaría comentar que hubiera estado bien incrementar más casos prácticos y poderlos 
desarrollar y discutir entre nosotros para tener más información sobre la realidad de las aulas en educación infantil. Así como comentar que la aportación de sistemas audiovisuales (vídeos, reportajes, documentales...) nos podría haber enriquecido más en las técnicas utilizadas en el aula".

Respecto a las críticas positivas, junto con los agradecimientos, son aportaciones generosas que permiten al profesorado reforzar su metodología didáctica y también mejorarla en este sentido.

Un ejemplo de crítica positiva es el siguiente: "Por último, considero que el calendario reflexivo ha sido una muy buena idea para que pudiéramos expresar posibles sugerencias, dudas o qué es lo que nos había aportado la sesión".

Un ejemplo de agradecimiento sería: "La asignatura de Lecto es muy interesante. Pienso que los temas finales han sido mucho más interesantes que los anteriores. Es decir, para mí son temas mucho más útiles para el día de mañana cuando sea maestra. Tu manera de trabajar en las clases es muy amena y productiva. Gracias".

Pocas muestras de autocrítica se han manifestado. Una de las más explícitas se refiere al trabajo en grupo y no al trabajo personal dentro del grupo: "Nuestro grupo de trabajo ya tuvo algunos problemas en la primera parte de la asignatura, pero, por suerte, conseguimos arreglarlos para poder continuar con el trabajo de la asignatura y llegar al objetivo que desaseábamos. Pero, en esta segunda parte, los problemas han aumentado. Por mi parte creo que intenté de todas las maneras posibles que el grupo funcionase, pero algunas compañeras no ponían de su parte y era imposible que hubiera la comunicación que habría deseado. De modo que me di cuenta que los trabajos que hacíamos y entregábamos no eran el resultado que yo hubiera deseado".

La necesidad de autocrítica como método mejora de los aprendizajes, a través de estrategias metacognitivas (Monereo y Castelló, 1997), también constituye uno de los temas a desarrollar dentro de los procesos reflexivos de los estudiantes.

La última categoría encontrada mínimamente ha sido la relación con otra teoría. Aunque conseguir la transversalidad es un objetivo muy valorado, se percibe que no se ha conseguido. El único ejemplo encontrado es el siguiente, que vincula la adquisición de la lectoescritura con teorías de la psicología del aprendizaje y con la $1^{\text {a }}$ parte de la Lectoescritura. "Me he tomado la libertad de añadir la palabra motivador a la teoría de Vigotsky de la Zona de desarrollo Próximo o a la teoría de Brunner del "Andamiaje". Esto se debe a la propia opinión sobre la motivación dentro y fuera del aula, que tiene que presentar un maestro creativo, ilusionado, implicado, observador, guía, soñador y receptivo, pero sobre todo motivador".

Se considera la transversalidad en los aprendizajes como uno de los elementos competenciales destacables para la formación de docentes responsables, por lo tanto, un nuevo componente como prospectiva para el diseño de la formación como aprendices reflexivos.

\section{CONCLUSIONES}

Los objetivos de esta primera fase de la investigación se concretaban en: 1. Evidenciar el conocimiento de los procesos de enseñanza y aprendizaje del lenguaje y la lectoescritura; 2. Argumentar de manera crítica el desarrollo del propio proceso de enseñanza y aprendizaje, y 3. Mostrar iniciativa para profundizar en los contenidos estudiados.

En relación al primer objetivo, se concluye que mayoritariamente los estudiantes vinculan el aprendizaje de los procesos de aprendizaje con la adquisición de contenidos, con lo que conviene reconstruir el concepto de reflexión para desvincularlo del concepto de resumen conceptual.

Sería conveniente contribuir a la mejora del concepto de práctica reflexiva con la incorporación de otros instrumentos que conduzcan a su delimitación. Por ejemplo, Salinas, Chandía y Rojas (2017) ofrecen la posibilidad de medir el constructo a través de 3 subescalas que valoran respectivamente la reflexión superficial, la pedagógica y la reflexión crítica. Los resultados se obtienen a través de un formato de autoinforme y por lo tanto adquieren un carácter simbólico en relación a la propia percepción del estudiante.

Referente al segundo objetivo, concluimos que los aspectos críticos mostrados en las reflexiones son poco significativos y que los estudiantes necesitan mejorar tanto el conocimiento como la argumentación del "cómo aprenden" para mejorarlos. Concretamente se considera necesario reafirmar los contenidos en relación a la transferencia de aprendizajes y a la autocrítica como medio para desarrollar la autorregulación.

Desde un punto de vista crítico para la mejora de los estudiantes, es deseable tratar aspectos como el autoconocimiento y la autorregulación. Por esta razón, se están realizando investigaciones (Vettraino y Linds, 2018) que determinan que también se construye autoconocimiento a través de la reflexión 
sobre las relaciones personales. Actividades interpretativas como el storytelling y la narrativa contribuyen a estos aprendizajes.

Es necesario trasladar a los estudiantes que tanto las críticas como las autocríticas no constituyen un elemento negativo. Al contrario, han de ser elementos que propicien la mejora. Deben conocer el desarrollo sistemático de estos procesos de crítica y autocrítica con la finalidad de conseguir este hábito de trabajo y de ejercicio de la sinceridad.

En relación al tercer objetivo, y vinculándolo con los objetivos anteriores, los hábitos de aprender a aprender se materializan con la voluntad de profundizar y ampliar los conocimientos. Se trataría de una acción como aprendiz estratégico para trasladarlo como docente estratégico (Monereo y Castelló, 1997) a sus estudiantes en un futuro profesional.

Como conclusión emergente, se determina que las reflexiones de los estudiantes de Educación Infantil en pocas ocasiones representan categorías puras, en relación al desarrollo de un solo valor, sino que se presentan a partir de la relación de varios conceptos. La más recurrente de las variaciones vincula el interés en la materia con la funcionalidad futura. Con ello deducimos que las materias más interesantes deberán tener un enfoque lo más práctico posible.

\section{PROSPECTIVA}

Este artículo presenta los resultados iniciales de una primera fase de la investigación. En un futuro, se pretenden analizar los resultados obtenidos en los portafolios de las demás asignaturas que forman parte de esta propuesta educativa y compararlos entre sí. Es posible que, mediante una comparativa de los resultados de las cuatro asignaturas, se pueda percibir la evolución de los estudiantes en relación a su comunicación y a las reflexiones que generan. Además, estos resultados también se podrán considerar en relación con la aplicación de otra innovación docente de estas asignaturas: el storytelling para la reflexión grupal. Este será un elemento clave para poder comparar sus reflexiones individuales con las grupales longitudinalmente. Además, también se podrán triangular con los resultados que van obteniendo de las asignaturas y estudiar posibles correlaciones. Así pues, los varios elementos reflexivos de estas cuatro materias merecen distintos estudios de cierto interés en el proceso reflexivo que desempeñan los futuros maestros en el aprendizaje de las lenguas y lectoescritura.

\section{REFERENCIAS}

Aloso Tapia, J., y Montero García-Celay, I. (2008). Orientación motivacional y estrategias motivadoras para el aprendizaje. A C. Coll, J. Palacios y Á. Marchesi, Desarrollo Psicológico y educación 2. Psicología de la educación escolar (pp. 259-305). Madrid: Alianza.

Bisquerra Alzina, R. (2004). Metodlogía de la investigación educativa. Madrid: La Muralla.

Bruner, J. (1997). La educación, puerta de la cultura. Madrid: Visor.

Domingo Roget, À. (2013). Pràctica reflexiva para docentes. de la reflexión ocasional a la reflexión metodológica. Saarbrücken: Publicia.

Gallardo-Paúls, B. (2008). Las huellas lingüísticas de la teoría de la mente: intersubjetividad y enunciación en el trastorno por déficit de atención/hiperactividad. Rev Neurol, 46, 29-35.

Huertas, J., Alonso Tapia, J., Trías, D., Ardura, A., y García, E. (2011). Los escenarios académicos, las identidades de los estudiantes universitarios y el movimiento y cambio de las orientaciones motivacionales entre ellos. A C. Monereo y J. Pozo, La identidad en psicología de la Educación (pp. 141- 153). Madrid: Narcea.

Le Fevre, D. (2010). Changing TACK: Talking about Change knowledge for professional learning. A H. Timperley, y J. Parr, Weaving evidence, inquiry and standards to build better schools (pp. 71-92). Wellington: NZCER.

Miras, M. (2008). Afectos, emociones, atribuciones y expectativas: el sentido del aprendizaje escolar. A C. Coll, J. Palacios, y A. Marchesi, Desarrollo psicológico y educaciúb 2. Psicología de la educación escolar (pp. 309-325). Madrid: Alianza.

Monereo, C., y Badia, A. (2011). los heterónimos del docente. Identidad, selfs y enseñanza. A C. Monereo y J. Poso, La identidad en psicología de la educación (pp. 5776). Madrid: Narcea.

Monereo, C., y Castelló, M. (1997). Las estrategias de aprendizaje. Cómo incorporarlas a la práctica educativa. Barcelona: Edebé.

Puig, J. (2009). Aprendizaje Servicio (ApS). Barcelona: Graó.

Rubio Hurtado, M., Galan Fernández, C., y Rodríguez Illera, J. (2013). EDUTEC. Revista Electrónica de Tecnología Educativa. Recuperado de http://edutec.rediris.es/Revelec2/Revelec43/propuesta _didactica_portafolios_digitales_educacion_superior.h tml

Salinas, Á., Chandía, E., y Rojas, D. (2017). Validación de un instrumento cuantitativo para medir la práctica reflexiva de docentes en formación. Estudios pedagógicos, 43(1) 289-309.

Schön, D. (1992). La formación de profesionales reflexivos. Hacia un nuevo diseño de la enseñanza y el aprendizaje de las reflexiones. Barcelona: Paidós.

Vettraino, E., y Linds, W. (2018). Living the experience of learning: embodied reflexivity as pedagogical process. SATJ: South African Theatre Journal, 31, 15-35.

Vilà Baños, R., y Aneas, A. (2013). Los seminarios de práctica reflexiva en el Practicum de Pedagogía de la Universidad de Barcelona. Bordón, 65(3), 165-181.

Vila Mendiburu, I. (1999). Jerome Bruner i l'adquisició del llenguatge. Temps d'Educació, 21, 77-90.

Vygotsky, L. (2012). Pensamiento y lenguaje. Barcelona: Paidós. 
Woollfolk, A., Hughes, M., y Walkup, V. (2008). Motivation in Learning and Teaching. A A. Woolfolk, M. Hughes, y

V. Walkup, Psychology in Education (pp. 437-480).

Harlow: Pearson. 